\title{
Influence of the Goos-Hänchen Shift on Tunneling Times in Dispersive Nonlinear Media
}

\author{
I. IliĆ ${ }^{a, *}$, P.P. BeličEV ${ }^{a}$, V. MilanoviĆ ${ }^{b}$, J. RadovanoviĆ ${ }^{b}$ And LJ. HadŽIevski ${ }^{a}$ \\ ${ }^{a}$ Vinča Institute of Nuclear Sciences, Belgrade, Belgrade Serbia \\ ${ }^{b}$ Faculty of Electrical Engineering, University of Belgrade, Belgrade, Serbia
}

\begin{abstract}
The Goos-Hänchen effect is an optical phenomenon defined as a displacement of the reflected beam of linearly polarized light after the total internal reflection, which occurs perpendicularly to the direction of propagation. Due to this effect, when an optical wave propagates through a barrier, tunneling times may change accordingly. In this paper, we consider the impact of the Goos-Hänchen effect on group delay and dwell time for electromagnetic wave propagating through a nonlinear dispersive slab placed inside linear dispersive surroundings. Numerical calculations are performed for the special case, namely a double negative index metamaterial embedded into a material with a saturable nonlinearity, when a background medium is vacuum. The numerical results for tunneling times are calculated when the Goos-Hänchen effect is observed. It is shown that this approach gives more accurate expressions for tunneling times when the angle of incidence has a non-zero value.
\end{abstract}

PACS numbers: 03.65.Xp, 42.25.Bs, 42.65.Hw, 78.20.Ci

\section{Introduction}

The well-known problem of tunneling from quantum mechanics can be transferred into the domain of classical electromagnetics, owing to the analogy between the Schrödinger and the Helmholtz equation. Significant research efforts have been invested lately in determining various time-related properties which characterize the electromagnetic (EM) wave propagation, such as tunneling times [1, 2]. While there have been several definitions offered for tunneling times, two of them seem to be the most appropriate - the dwell time and the group delay [3]. The dwell time is defined via an integrated probability or stored energy, while the group delay can be found from the frequency derivative of the transmission phase shift. In case of EM propagation, tunneling times were first calculated by Winful [4]. The recent papers provided the analysis of dwell time and group delay for all linear media [5], the media with Kerr and saturable type of nonlinearity $[6,7]$ as well as the media comprising left-handed metamaterials (LHMs) embedded into a dielectric with Kerr or saturable type of nonlinearity [8], but the Goos-Hänchen effect has been neglected in these calculations. This influence of the Goos-Hänchen shift on tunneling times, i.e. group delay and dwell time, has been previously investigated for a barrier made of a linear nondispersive material [9].

When an EM wave is totally reflected it experiences a lateral displacement from its position predicted by geometric optics because each of its plane-wave components

\footnotetext{
* corresponding author; e-mail: igori@vinca.rs
}

undergoes a different phase change [10]. This effect is known as the Goos-Hänchen shift and it has been studied in numerous optical systems, such as LHMs [11], Otto configuration [12], metal surfaces [13], and so on. While the Goos-Hänchen shift is usually much smaller than the beam width, in layered systems supporting surface waves, this shift can be larger [14]. This situation is observed at the interface between right and left-handed materials, where the surface polaritons occur and dramatically enhance the Goos-Hänchen effect because of their possibility to transfer the energy along the interface [15].

The inverse Snell law, reversed Doppler effect and Cerenkov backward radiation are consequences of the negative index of refraction $\left(n_{\mathrm{R}}=\Re(\sqrt{\varepsilon \mu})\right)$, which was predicted by Veselago in 1967 [16]. Metamaterials exhibiting this property, called LHMs, were first demonstrated by Smith et al. in 2000 [17]. The combination of a periodic array of metal wires with regular array of split-ring resonators (SRRs) exhibited negative refractive index in the microwave range of frequencies. Recent papers $[18,19]$ have verified the existence of left-handed characteristics in $\mathrm{THz}$ range, as well.

The nonlinear wave propagation in LHMs was observed and analyzed for two cases: embedding SRRs and wires into a Kerr-type nonlinear dielectric [20], or inserting certain nonlinear elements in each SRR slit [21]. In both situations, dielectric permittivity $\varepsilon$ and magnetic permeability $\mu$ were functions of not only the incident field frequency, but of its intensity as well. Similar observations were made for nonlinear photorefractive crystals $\left(\mathrm{GaAs}, \mathrm{LiNbO}_{3}\right)$, which possess saturable type of nonlinearity [22]. 
In this paper, we analyze the influence of the GoosHänchen shift on tunneling times for EM waves (TE modes) propagating through a barrier made of LHM embedded into a dielectric with saturable or Kerr type of nonlinearity.

\section{Theoretical consideration}

Consider a barrier with thickness $L$, placed inside a planar linear waveguide which is characterized by parameters, $\varepsilon_{b}$ and $\mu_{b}$ (Fig. 1). The barrier is made of LHM with metal wires and SRRs, embedded into a dielectric with saturable nonlinearity. Since its permittivity is a function of incident field frequency and intensity, and its permeability depends only on $\omega$, these parameters can be described by

$$
\begin{aligned}
\varepsilon_{s} & =\varepsilon_{\mathrm{LHM}}+\varepsilon_{\mathrm{NL}}=\varepsilon_{\infty}\left(1-\frac{\omega_{\mathrm{p}}^{2}}{\omega^{2}-\omega_{\mathrm{r}}^{2}+\mathrm{i} \Gamma_{\mathrm{e}} \omega}\right) \\
& +\varepsilon_{\mathrm{NL}}, \\
\mu_{s} & =1-\frac{F \omega_{0}^{2}}{\omega^{2}-\omega_{0}^{2}+\mathrm{i} \Gamma_{\mathrm{m}} \omega} .
\end{aligned}
$$

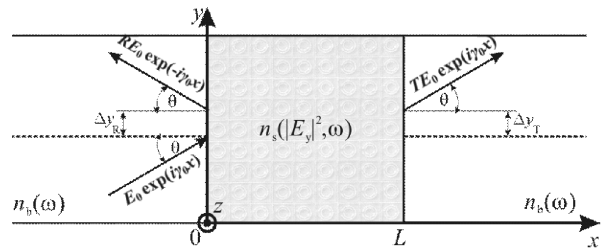

Fig. 1. The model.

Here, $\varepsilon_{\infty}$ is the background dielectric constant, $\omega_{\mathrm{p}}$ is the plasmafrequency-measure of the strength of interaction between the oscillators and the electric field, $\omega$ is the frequency of incident EM wave, $\omega_{\mathrm{r}}$ is the resonance frequency of the electric dipole oscillators, while $\omega_{0}$ is the resonance frequency of the magnetic dipole oscillators, $\Gamma_{\mathrm{e}}$ and $\Gamma_{\mathrm{m}}$ stand for the damping frequencies of the electric and magnetic fields, respectively, $F$ is a measure of the strength of the interaction between the oscillators and magnetic field and $\varepsilon_{\mathrm{NL}}=\varepsilon_{\mathrm{NL}}\left(\left|E_{y}\right|^{2}\right)$ represents the intensity-dependent part of permittivity, and is given by [23]:

$$
\varepsilon_{\mathrm{NL}}=\varepsilon_{\mathrm{D} 0}+\alpha \frac{\left|E_{y}\right|^{2} / E_{\mathrm{c}}^{2}}{1+\kappa\left|E_{y}\right|^{2} / E_{\mathrm{c}}^{2}} .
$$

Here, $\varepsilon_{\mathrm{D} 0}$ is the linear dielectric permittivity, $E_{\mathrm{c}}$ is the characteristic electric field, $\alpha= \pm 1$ is the sign of nonlinearity, i.e. $\alpha=1$ for focusing and $\alpha=-1$ for defocusing nonlinearity, and $\kappa$ is the saturation strength. The second term of the permittivity is a nonlinear function of the electric field intensity and describes saturable type of nonlinearity and will be noted as $\varepsilon_{\mathrm{SNL}}$. We presume that $\alpha \neq \alpha(\omega), E_{\mathrm{c}} \neq E_{\mathrm{c}}(\omega)$ and $\kappa \neq \kappa(\omega)$.

The Goos-Hänchen shift does not alter the dwell time, since it is defined as an interaction time in terms of steady-state problems and without regard for the particular scattering channel (reflection or transmission) taken by a given photon.

Presume that the barrier is irradiated with a transverse electric (TE) wave along $x$ axis. As can be seen from Fig. 1, due to the Goos-Hänchen effect the EM wave experiences lateral shifts after its interaction with interfaces at the beginning $(x=0)$ and at the end of the barrier $(x=L)$.

According to the paper by Steinberg and Chiao [9], total phase of the electric field at the end of the barrier $(x=L)$ in this model, can be written as

$$
\varphi_{\text {tot }}(L)=\varphi_{0}+k_{y} \Delta y_{\mathrm{T}}-\omega t,
$$

where $k_{y}=(\omega / c) \sqrt{\varepsilon_{b} \mu_{b}} \sin \theta$ is the $y$ component of the wave vector, $\Delta y_{\mathrm{T}}$ stands for the lateral shift of EM wave, $\theta$ is the incident angle, and $\varphi_{0}=\varphi_{\mathrm{T}}+k_{x} L$ represents the phase of the incident wave. Here, $\varphi_{\mathrm{T}}$ stands for the phase of transmittivity $T$, while $k_{x}=(\omega / c) \sqrt{\varepsilon_{b} \mu_{b}} \cos \theta$ is the $x$ component of the wave vector.

Assuming the stationary-phase approximation and the paraxial approximation, we can write [9]:

$$
\begin{aligned}
& \left.\left(\frac{\mathrm{d} \varphi_{\text {tot }}}{\mathrm{d} \omega}\right)\right|_{\theta}=0, \\
& \left.\left(\frac{\mathrm{d} \varphi_{\text {tot }}}{\mathrm{d} \theta}\right)\right|_{\omega}=0 .
\end{aligned}
$$

Inserting the expression (4) for the total phase into Eq. (5) leads to

$$
\begin{gathered}
\tau_{\mathrm{g} T 0}+\left[\frac{\sqrt{\varepsilon_{b} \mu_{b}}}{c} \sin \theta+\frac{\omega}{c} \sin \theta \frac{\partial \sqrt{\varepsilon_{b} \mu_{b}}}{\partial \omega}\right] \Delta y_{\mathrm{T}} \\
-\tau_{\mathrm{g} T}=0,
\end{gathered}
$$

where $\tau_{\mathrm{g} T 0}=\left.\frac{\partial \varphi_{0}}{\partial \omega}\right|_{\theta}$ stands for the group delay of transmitted part of the EM wave without the Goos-Hänchen shift.

The lateral shift $\Delta y_{\mathrm{T}}$ can be obtained by inserting (4) into (6), which gives

$$
\Delta y_{\mathrm{T}}=-\frac{1}{k_{0} \cos \theta \sqrt{\varepsilon_{b} \mu_{b}}} \frac{\partial \varphi_{0}}{\partial \theta},
$$

where $k_{0}$ stands for the wave vector in vacuum. Combining the expressions (8) and (7), we find the group delay of the transmitted part of EM wave in the form

$$
\tau_{\mathrm{g} T}=\tau_{\mathrm{g} T 0}-\frac{\tan \theta}{\omega}\left(\frac{\partial \varphi_{0}}{\partial \theta}\right)\left[1+\frac{\omega}{\sqrt{\varepsilon_{b} \mu_{b}}} \frac{\partial \sqrt{\varepsilon_{b} \mu_{b}}}{\partial \omega}\right] .
$$

Analogously, we obtain the expression for the group delay for the reflected EM wave

$$
\tau_{\mathrm{g} R}=\tau_{\mathrm{g} R 0}-\frac{\tan \theta}{\omega} \frac{\partial \varphi_{\mathrm{R}}}{\partial \theta}\left[1+\frac{\omega}{\sqrt{\varepsilon_{b} \mu_{b}}} \frac{\partial \sqrt{\varepsilon_{b} \mu_{b}}}{\partial \omega}\right],
$$

where $\varphi_{\mathrm{R}}$ is the phase of the reflectivity, and $\tau_{\mathrm{g} R 0}=$ $\left.\frac{\partial \varphi_{\mathrm{R}}}{\partial \omega}\right|_{\theta}$ represents the group delay of the reflected part of EM wave in the absence of the Goos-Hänchen shift.

Now, the total group delay can be calculated as

$$
\tau_{\mathrm{g}}=|T|^{2} \tau_{\mathrm{g} T}+|R|^{2} \tau_{\mathrm{g} R} .
$$

For an incident angle $\theta$ equal to the critical angle of the total reflection $\theta_{\mathrm{CR}}$, the dwell time diverges, while the group delay remains finite. This can be explained by 
the fact that in this case, the refracted wave is almost parallel to the interfaces of the barrier, which implies infinite dwell time. On the other hand, group delay is finite, since the bounded beam undergoes diffraction and begins to couple out of the barrier sooner than predicted by ray optics.

\section{Numerical results}

The numerical results are obtained for the LHM barrier with the self-defocusing nonlinear medium $(\alpha=$ $-1)$ placed inside the vacuum $\left(\varepsilon_{b}, \mu_{b}=1\right)$ with parameters: $\varepsilon_{\infty}=3.1, \omega_{\mathrm{p}}=2700 \mathrm{THz}, \omega_{\mathrm{r}}=0, \Gamma_{\mathrm{e}}=$ $35 \mathrm{THz}, F=0.052, \omega_{0}=2300 \mathrm{THz}, \Gamma_{\mathrm{m}}=35 \mathrm{THz}$, $E_{\mathrm{c}}=1 \mathrm{~V} / \mathrm{m}, \kappa=1, L=100 \mathrm{~nm}$ and $\varepsilon_{\mathrm{D} 0}=2$. The effective refractive index of the barrier is defined by $n_{\text {eff }}=(1 / L) \int_{0}^{\mathrm{L}} \Re(n(x)) \mathrm{d} x$. For the applied incident field with the amplitude $E_{0}=15 \mathrm{~V} / \mathrm{m}$, the barrier exhibits a negative refractive index in the frequency range of $2254 \mathrm{THz} \leq \omega \leq 2348 \mathrm{THz}$.

For the incident angle $\theta=\pi / 4$, the EM wave experiences the Goos-Hänchen shift, which causes significant increase in the group delay outside the frequency range for which the barrier has negative refractive index (Fig. 2). However, in the region of a negative refractive index, the Goos-Hänchen shift is negligible. Therefore, one can conclude that the Goos-Hänchen shift practically vanishes in slabs where negative refractive index conditions are achieved.

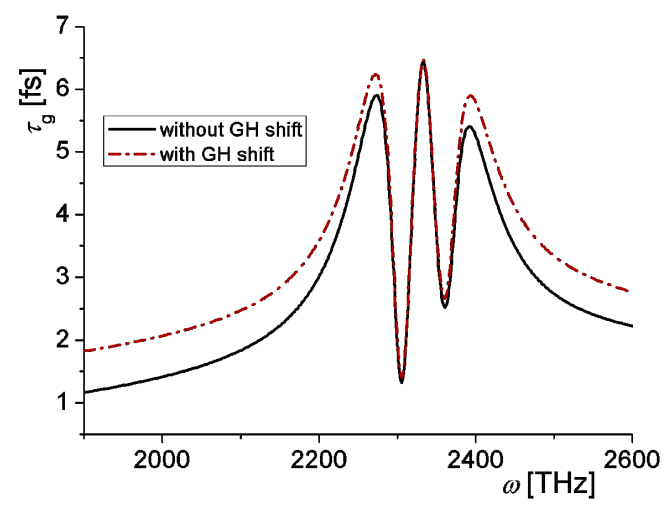

Fig. 2. Group delay versus incident wave frequency in case of the Goos-Hänchen shift included and the GoosHänchen shift excluded for incident angle $\theta=\pi / 4$.

In order to validate this assumption, results for the dependence of the group delay on the incident angle are obtained for two different frequencies, $\omega_{1}=2272 \mathrm{THz}$ and $\omega_{2}=2300 \mathrm{THz}$ (Fig. 3). The first frequency, $\omega_{1}$, is chosen at the boundary of the frequency region where refractive index is negative $\left(n_{\text {eff }}=-0.05\right)$, while the second frequency $\omega_{2}$ is at the center of that region $\left(n_{\mathrm{eff}}=-0.34\right)$. Although some the Goos-Hänchen shift still exists, these results are in a good agreement with the previous conclusion. Also, for small incident angles, the Goos-Hänchen

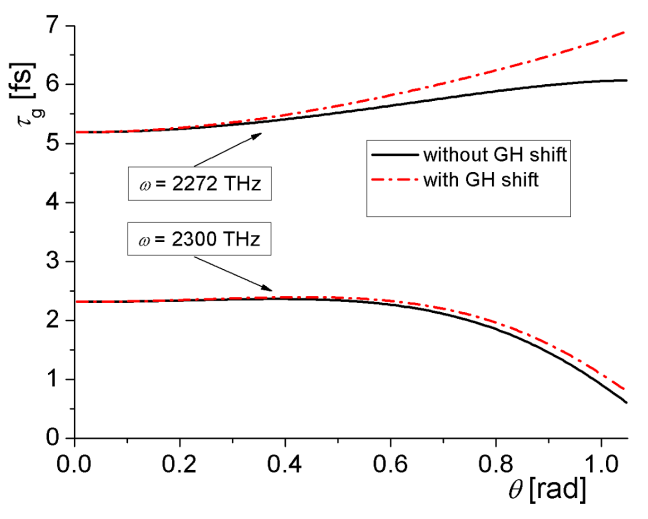

Fig. 3. Dependence of the group delay on incident angle in case of the Goos-Hänchen shift included and the Goos-Hänchen shift excluded for two different incident wave frequencies: $\omega_{1}=2272 \mathrm{THz}$ and $\omega_{2}=2300 \mathrm{THz}$.

shift is negligible for all incident field frequencies, as expected. It should also be mentioned that the reflected and transmitted the Goos-Hänchen shifts, $\Delta y_{\mathrm{R}}$ and $\Delta y_{\mathrm{T}}$, are equal for the observed model.

\section{Conclusion}

The influence of the Goos-Hänchen shift on the tunneling times in case of EM wave propagating through a barrier made of SRRs and long metal wires embedded in material with saturable nonlinearity is observed, and corresponding expressions are derived. It is demonstrated that this phenomenon has different effects on various types of tunneling times. The Goos-Hänchen shift has no influence on the dwell time but strongly impacts the group delay. The numerical results revealed that, for a barrier with negative index of refraction within a particular range of frequencies, the Goos-Hänchen effect can be significantly reduced by modifying the incident wave frequency and shifting it towards the center of this negative refractive index range.

\section{References}

[1] A. Enders, G. Nimtz, J. Phys. I (France) 2, 1693 (1992).

[2] A.M. Steinberg, P.G. Kwiat, R.Y. Chiao, Phys. Rev. Lett. 71, 708 (1993).

[3] E.H. Hauge, J.A. Støvneng, Rev. Mod. Phys. 61, 917 (1989).

[4] H.G. Winful, Phys. Rev. E 68, 016615 (2003).

[5] I. Ilić, P.P. Beličev, V. Milanović, J. Radovanović, J. Opt. Soc. Am. B 25, 1800 (2008).

[6] P.P. Beličev, I. Ilić, J. Radovanović, V. Milanović, Lj. Hadžievski, Acta Phys. Pol. A 115, 834 (2009).

[7] G. Isić, V. Milanović, J. Radovanović, Z. Ikonić, D. Indjin, P. Harrison, Phys. Rev. A 77, 033821 (2008).

[8] P.P. Beličev, I. Ilić, V. Milanović, J. Radovanović, Lj. Hadžievski, Phys. Rev. A 80, 023821 (2009). 
[9] A.M. Steinberg, R.Y. Chiao, Phys. Rev. A 49, 3283 (1994).

[10] F. Goos, H. Hänchen, Ann. Phys. 1, 333 (1947).

[11] P.R. Berman, Phys. Rev. E 66, 067603 (2002).

[12] I.V. Shadrivov, A.A. Zharov, Y.S. Kivshar, Appl. Phys. Lett. 83, 2713 (2003).

[13] P.T. Leung, C.W. Chen, H.P. Chiang, Opt. Commun. 276, 206 (2007).

[14] S. Chuang, J. Opt. Soc. Am. A 3, 593 (1986).

[15] R. Ruppin, Phys. Lett. A 277, 61 (2000).

[16] V.G. Veselago, Sov. Phys. Usp. 10, 509 (1967).

[17] D.R. Smith, W. Padilla, D.C. Vier, S.C. Nemat-Nasser, S. Shultz, Phys. Rev. Lett. 84, 4184 (2000).

[18] U.K. Chettiar, A.V. Kildishev, H.-K. Yuan, W. Cai, S. Xiao, V.P. Drachev, V.M. Shalaev, Opt. Lett. 32 1671 (2007)
[19] A.N. Grigorenko, A.K. Geim, H.F. Gleeson, Y. Zhang, A.A. Firsov, I.Y. Khrushchev, J. Petrović, Nature (London) 438, 335 (2005).

[20] A.A. Zharov, I.V. Shadrivov, Y. Kivshar, Phys. Rev. Lett. 91, 037401 (2003).

[21] M. Lapine, M. Gorkunov, K.H. Ringhofer, Phys. Rev. E 67, 065601 (2003).

[22] W.J. Padilla, A.J. Taylor, C. Highstrete, M. Lee, R.D. Averitt, Phys. Rev. Lett. 96, 107401 (2006).

[23] A. Maluckov, Lj. Hadžievski, N. Lazarides, G.P. Tsironis, Phys. Rev. E 77, 046607 (2008). 